\section{Recognizing Pseudocholinesterase Deficiency in the Post-operative Patient: Diagnosis and Management in the ICU}

Jared A. Ojile, M.D., Ashlie A. Elver, B.S.

University of Kansas School of Medicine-Wichita, Wichita, KS

Department of Internal Medicine

Received Feb. 7, 2021; Accepted for publication March 30, 2021; Published online July 9, 2021 https: doi.org 10.17161 lijm.voll41.5169

\section{INTRODUCTION}

Succinylcholine is a paralytic drug commonly used during general surgery. ${ }^{1}$ It is a depolarizing paralytic, meaning that it achieves its paralytic effect by overstimulating the neuromuscular endplate and thereby blocking neuromuscular junction-released acetylcholine from causing muscular contraction. The onset of action is typically within seconds of injection and usually declines and clears over a course of a few minutes. Clearance of the drug is dependent on the enzyme pseudocholinesterase, which degrades succinylcholine. Genetic or acquired deficiency of pseudocholinesterase therefore results in unexpected prolongation of succinylcholine clearance and prolonged flaccid paralysis.

Management of pseudocholinesterase deficiency centers on re-initiating or maintaining the patient's intubated status until the patient's ability to draw proper tidal volumes spontaneously returns as the drug is slowly cleared. ${ }^{2}$ Depending on whether the patient is heterozygous or homozygous for the genetic enzyme deficiency, the duration of extended flaccid paralysis can last up to a few hours. Acquired forms of pseudocholinesterase deficiency can occur during trauma, organ failure, malignancy, certain medication, and other metabolically demanding disease states, but are not discussed in detail here.

\section{CASE REPORT}

A 53-year-old male presented to the intensive care unit (ICU) directly from the operating room due to delay of extubation. Medications used for general anesthesia included the following systemic drugs: midazolam $2 \mathrm{mg}$, fentanyl $250 \mathrm{mcg}$, lidocaine $100 \mathrm{mg}$, and propofol $540 \mathrm{mg}$. Surgical paralysis was achieved using succinylcholine $120 \mathrm{mg}$ and rocuronium $10 \mathrm{mg}$. Other medications given intraoperatively and perioperatively included cefazolin $2000 \mathrm{mg}$, ephedrine $80 \mathrm{mg}$, ondansetron $4 \mathrm{mg}$, abuterol HFA inhaler 8 puffs, hydromorphone $1 \mathrm{mg}$, and $1500 \mathrm{ml}$ of crystalloid fluids.

The surgery was without complication. Upon completion of the procedure, the patient failed to meet criteria for extubation. Per the anesthesiologist's description, the patient was highly tachypneic, but failed to draw appropriate tidal volumes while on appropriate pressure support. Sugammadex was given to reverse rocuronium blockade but had no effect. He was transferred to the intensive care unit while still intubated for further monitoring. Labs obtained upon transferring, including blood counts and electrolytes, were unremarkable. Approximately three hours later the patient passed a spontaneous breathing trial and was extubated successfully. The patient required no further oxygen supplementation. He was able to reposition himself in his hospital bed with ease shortly after extubation, limited only by expected post-operative pain.

The patient's past medical history was significant for recovery from opioid use disorder. He received methadone $80 \mathrm{mg}$ PO daily from the

\section{KANSAS JOURNAL of MEDIC INE}

local methadone clinic. Of note, he did not take his daily dose on day of surgery as instructed but was given $250 \mathrm{mcg}$ of fentanyl during the surgery. He also reported being diagnosed with Guillain-Barre Syndrome in 2006 and was treated on multiple occasions over subsequent years with IVIG, plasmapheresis, and oral steroids. He ultimately received the diagnosis of chronic inflammatory demyelinating polyneuropathy in 2013 and took neuropathic pain agents daily.

The patient has an otherwise unremarkable surgical history. He previously had undergone arthroscopy of the knee at an outside hospital under local anesthesia presumably without the use of systemic paralytics. After the patient had an opportunity to talk to his family, further interview revealed an event where he was in a "coma” following a procedure as an infant. He learned from his sister that she also had recently experienced similar prolonged paralysis during a hysterectomy and had been diagnosed with presumed pseudocholinesterase deficiency. The patient had eight children with unknown surgical and anesthesia histories.

\section{DISCUSSION}

Since genetic analysis of this patient was unfeasible due to the resources available, pseudocholinesterase deficiency was an inclusion and exclusion clinical diagnosis. Due to the patient's history of opiate abuse, current methadone use, and history of Guillain-Barre Syndrome, it is important to differentiate how each of these and other neuromuscular etiologies would present and how they differed from the patient's case. The anesthesiologist noticed that while the patient was on appropriate pressure support before extubation, he was tachypneic and failed to draw proper tidal volumes. It is unclear whether the patient was fully alert at this time, but upon arrival to the ICU on ventilatory support approximately 20 minutes later, the patient was able to follow basic movement commands consistently and answer basic questions with nods and shakes of head.

The first possibility ruled out was opioid-induced respiratory depression. The patient took $80 \mathrm{mg}$ of methadone per day, which he was instructed to hold on day of surgery. No information to the contrary indicated that the patient was noncompliant with this instruction. During the procedure, the patient received $250 \mathrm{mcg}$ of fentanyl. The team was confident in ruling out narcotic toxicity as the etiology due to the patient's tachypnea, which indicated an intact central respiratory drive. While opioid toxicity can cause poor tidal volumes due to opioid binding patterns in the pons, it would be unusual for a patient to have compensatory tachypnea in this kind of toxicity. ${ }^{3}$ Because of lack of evidence of opioid toxicity, naloxone was not attempted at this time.

Myasthenia gravis and myasthenic crisis are conditions that could present with a similar symptom profile as our patient. While multiple forms of myasthenia gravis exist based on the type of autoantibodies produced, the most common are those directed against the acetylcholine receptor $(\mathrm{AChR})$ on the neuromuscular endplate. ${ }^{4}$ These autoantibodies produce flaccid paralysis by blocking true acetylcholine binding as well as by degrading these receptors that are necessary for 
KANSAS JOURNAL of MEDICINE PSEDUOCHOLINESTERASE DEFICIENCY

continued.

neuromuscular signal translation. This is similar to the action of succinylcholine with one important distinction. While the autoantibodies of myasthenia gravis bind AChR on the neuromuscular endplate, they do not activate the receptor but instead block and degrade it. While succinylcholine binds and blocks acetylcholine receptors, it also activates these receptors repeatedly. This repetitive stimulation initially causes a fasciculation-induced paralysis followed by true flaccid paralysis. Unlike in myasthenia gravis, acetylcholinesterase inhibitors do not produce increased levels of true muscular contractions, but rather prolong succinylcholine's paralytic effect. Acetylcholinesterase inhibitors, therefore, cannot be used as an antidote when pseudocholinesterase levels are deficient. $^{5}$

Myasthenic crisis, which involves severe respiratory compromise as the muscles of respiration become involved in the myasthenic process, mimics our patient case very closely. As noted by the anesthesiologist, the patient appeared to have an intact respiratory drive, as he was tachypneic and in distress, but his poor tidal volumes indicated poor respiratory muscle capacity. Surgical procedures are known precipitants of myasthenic crisis. While management of myasthenic crisis can include IVIG and plasma exchange, a mainstay of management for both myasthenic crisis and pseudocholinesterase deficiency resulting in respiratory compromise is intubation with mechanical ventilation. Myasthenic crisis was low on our differential diagnosis given the patient's lack of history of myasthenia gravis, as well as his spontaneous full recovery within hours of the initial event. In either scenario, empiric acetylcholinesterase inhibitor use should be avoided given that these medications are contraindicated in both myasthenic crisis (as they can cause overproduction of lung secretions in a vulnerable patient) ${ }^{6}$ and pseudocholinesterase deficiency (as this will prolong the paralysis induced by succinylcholine) ${ }^{5}$

The patient's history of Guillain-Barre Syndrome (GBS)/Chronic Inflammatory Demyelinating Polyneuropathy was a factor to consider, especially given the relatively prolonged nature of his clinical course with that disease. The history of GBS was not discovered until after patient had recovered. Guillain-Barre was ruled out for several reasons. First, Guillain-Barre's clinical course usually extends over multiple weeks. The hyperacuity of the patient's symptoms, with him being neurologically intact before the procedure and complete neural recovery within hours indicated an etiology other than GBS. While GBS can produce respiratory distress through diaphragmatic involvement that would mimic his clinical symptoms, it would be unusual for a GBS patient not to have previously experienced other non-diaphragmatic muscle weakness as well. As mentioned previously, the patient was grossly neurologically intact before his surgical procedure. He was able to follow basic movement commands while on a ventilator but appeared diffusely weak rather than the ascending flaccid paralysis typical of GBS. ${ }^{7}$ Once extubated, he had full function of all muscles return equally, limited only by expected post-operative pain.

Because the patient received succinylcholine and rocuronium for intraoperative paralysis, the possibility of rocuronium toxicity had to be ruled out. Sugammadex is the preferred antidote for rocuronium toxicity. ${ }^{8}$ While still in the operating room, the patient was administered 100 mg sugammadex without effect. Given these factors, it was determined that pseudocholinesterase deficiency was the most likely etiology of the patient's compromised status. This diagnosis was supported by the patient's spontaneous recovery within hours.

Inherited pseudocholinesterase deficiency severity depends on the homozygosity vs. heterozygosity of the patient. Heterozygotes for the deficient enzyme occur in about 1 in 500 persons, and homozygotes for the deficient enzyme occur in about 1 in 2,000 - 5,000 persons. ${ }^{2}$ Homozygotes have more prolonged paralysis with succinylcholine than heterozygotes. Since genetic analysis of each pre-surgical patient is unfeasible and the genetic variant resulting in deficiency of pseudocholinesterase is relatively common, the surgical and anesthesia team must be on the lookout for this possibility. Preoperative screening should include a family history of delayed extubation. While this mutation is of less importance in the intraoperative phase due to the patient having continuous respiratory support, it is of supreme importance post-operatively, as the surfacing patient can begin to experience severe respiratory distress and accompanying mental terror. This requires immediate recognition, continuation of respiratory support, and possible mild sedation for patient comfort. Understanding the differential diagnosis and pathophysiology related to different causes of neuromuscular respiratory failure are critical to the ICU physician, as failure to recognize the correct etiology and administer the appropriate drug could harm the patient further.

\section{REFERENCES}

${ }^{1}$ Hager HH, Burns B. Succinylcholine Chloride. November 16, 2020. In: StatPearls [Internet]. Treasure Island, FL: StatPearls Publishing, 2020. PMID: 29763160

2 Trujillo R, West WP. Pseudocholinesterase Deficiency. July 15, 2020. In: StatPearls [Internet]. Treasure Island, FL: StatPearls Publishing, 2020. PMID: 31082076.

3 Dahan A, Aarts L, Smith TW. Incidence, reversal, and prevention of opioid-induced respiratory depression. Anesthesiology 2010; 112(1):226-238. PMID: 20010421

${ }^{4}$ Huijbers MG, Lipka AF, Plomp JJ, Niks EH, van der Maarel SM, Verschuuren, JJ. Pathogenic immune mechanisms at the neuromuscular synapse: The role of specific antibody-binding epitopes in myasthenia gravis. J Intern Med 2014; 275(1):12-26. PMID: 24215230.

${ }^{5}$ U.S. National Library of Medicine.ANECTINE ${ }^{\circledast}$ (Succinylcholine Chloride Injection, USP): A short-acting depolarizing skeletal muscle relaxant. Civica, Inc. July 2020. https://dailymed.nlm.nih.gov/dailymed/fda/fdaDrugXsl. cfm?setid=0b990f84-9515-44bd-a97d-a97bbead065c\&type=display . Accessed February 20, 2021

6 Wendell LC, Levine JM. Myasthenic crisis. Neurohospitalist 2011; 1(1):1622. PMID: 23983833

${ }^{7}$ Arcila-Londono X, Lewis RA. Guillain-Barré syndrome. Sem Neurol 2012; 32(03):179-186. PMID: 23117942.

8 Wu X, Oerding H, Liu J, et al. Rocuronium blockade reversal with sugammadex vs. neostigmine: Randomized study in Chinese and Caucasian subjects. BMC Anesthesiol 2014; 14:53. PMID: 25187755.

Keywords: pseudocholinesterase deficiency, airway extubation, intensive care unit 